\title{
Where will efficient energy release occur in 3D magnetic configurations?
}

\author{
P. Démoulin \\ Observatoire de Paris, section de Meudon, LESIA, UMR 8109 (CNRS), F-92195 \\ Meudon Cedex, France
}

\begin{abstract}
The energy needed to power flares is thought to be stored in the coronal magnetic field. However, the energy release is efficient only at very small scales. Magnetic configurations with a complex topology, i.e. with separatrices, are the most obvious configurations where current sheets can form, and then, reconnection can efficiently occur. This has been confirmed for several flares computing the coronal field and comparing the locations of the flare loops and ribbons to the deduced 3D magnetic topology. However, this view is too restrictive taking into account the variety of observed solar flaring configurations. Indeed, "Quasi-Separatrix Layers" (QSLs), which are regions where there is a drastic change in field-line linkage, generalize the definition of separatrices. They let us understand where reconnection occurs in a broader variety of flares than separatrices do. The strongest electric field and current are generated at, or close to where the QSLs are thinnest. This defines the region where particle acceleration can efficiently occur. A new feature of 3-D reconnection is the natural presence of fast field line slippage along the QSLs, a process called "slip-running reconnection". This is a plausible origin for the motions of the X-ray sources along flare ribbons.
\end{abstract}

Key words: Magnetic Reconnection, Sun: magnetic fields, Sun: X-rays, Sun: flares

\section{Introduction}

The solar corona is formed by a low- $\beta$ plasma where the magnetic field has a key role. One of the best examples are flares, where a large amount of magnetic energy is released, and the creation of new magnetic connectivities can be directly seen as flare loops. The progression of flare ribbons traces the displacement of the energy released location in the magnetic configuration (the ribbons are the mapping image, along field lines, of the energy release in the corona). However, the observations provide mostly informations on the 
consequences of magnetic energy release (as emission of heated plasma and radiation from accelerated particles, with small delays due to the energy transport processes). An important investigation work is needed to assemble all the observational facts to constrain the energy release mechanism.

It is striking to see, at a given time in a flare, how localized are the traces of energy release (ribbons, new flare loops). This shows that special conditions are needed to transform the stored magnetic energy. Indeed, there is an expected very low dissipation of the coronal field at the spatial scales of the global flaring configuration (typically an active region). The resistive term in the induction equation can become large enough only if narrow current layers are created. This is the origin of the spatial localization of the energy release in flares.

Current sheets can form easily along separatrices both in 2-D and 3-D configurations. The concept of separatrices has been generalized in 3-D configurations to QSLs and the theory was successfully tested with multi-wavelength observations of flares and less energetic events (Section 2). Why and how current layers are formed along Quasi-Separatrix Layers (QSLs) is summarized in Section 3. Then, the recent developments of 3-D magnetic reconnection are summarized, and set in the perspective of X-ray observations (Section 4). Finally, some of the remaining challenges theory has to face are discussed in Section 5.

\section{Separatrices and QSLs}

Separatrices are magnetic surfaces where the magnetic field line linkage is discontinuous. The simplest example is a 2-D magnetic configuration with an $\mathrm{X}$-point or null point (where the magnetic field vanishes). Two separatrices cross at the X-point, and they define four separated regions, called connectivity domains, where the magnetic connectivity changes continuously from one field line to its neighbor, but discontinously at the boundaries of the domains (separatrices).

The above 2-D concepts have been generalized to 3-D null points. The vicinity of a null point is described by the linear term in the local Taylor expansion of the magnetic field. The diagonalisation of the field's Jacobian matrix provides three orthogonal eigenvectors (Molodenskii and Syrovatskii, 1977). The divergence-free condition imposes that the sum of the three eigenvalues vanishes $\left(\lambda_{1}+\lambda_{2}+\lambda_{3}=0\right)$, and for magnetic fields in equilibrium with a plasma $(\vec{j} \times \vec{B}=\vec{\nabla} P)$ the eigenvalues are real (Lau and Finn, 1990). Then two eigenvalues, say $\lambda_{1}, \lambda_{2}$, have the same sign, opposite to the one of the third eigenvalue $\lambda_{3}$. The two eigenvalue field lines, which start from an infinitesimal distance of the null in directions parallel and anti-parallel to the eigenvector associated to $\lambda_{3}$, are called $\gamma$-lines, or spines. The separatrix, 
called $\Sigma$-surface or fan, is defined by all the field lines starting at an infinitesimal distance of the null in the plane defined by the two eigenvectors associated to $\lambda_{1}$ and $\lambda_{2}$. The $\gamma$-lines/spines and $\Sigma$-surface/fan are defined by the local properties of the null, but they have large scale implications for the topology of the magnetic field.

Two types of 3-D nulls exist depending on the sign of the $\Sigma$-surface/fan eigenvalues; their sign is used to define the sign of the null. A more complex topology is build with two "interacting" nulls of opposite signs, called A and B. The intersection of the fans $\left(\Sigma_{A}\right.$ and $\left.\Sigma_{B}\right)$ define a magnetic null-null line or separator. The separatrices of the configuration are the two surfaces $\Sigma_{A}$ and $\Sigma_{B}$ bounded by the $\gamma$-lines (giving the possibility to have unclosed separatrices, Lau, 1993). The complexity of the field topology increases rapidly with the number of nulls present. Longcope and Klapper (2002) provided a general theoretical framework to analyze the frequently very complex magnetic topology of 3-D configurations formed by many flux tubes (as observed in magnetograms).

An important location for current-sheet formation, then, for reconnection in a classical view, is the intersection of two separatrices, which is a null point in 2-D and a separator in 3-D (Gorbachev and Somov, 1988; Lau and Finn, 1990; Longcope and Cowley, 1996). More generally, current sheets are thought to form all along the separatrices when arbitrary motions are imposed at their intersection with the lower line-tied boundary (e.g. Zwingmann et al., 1985; Low and Wolfson, 1988; Aly, 1990; Lau, 1993; Yokoyama and Shibata, 1994). For further information see the review of Longcope (2005).

Some of the studied flaring active regions (ARs) have indeed, at least, one magnetic null point in the extrapolated coronal fields (e.g. Mandrini et al., 1993; Gaizauskas et al., 1998; Longcope and Silva, 1998; Aulanier et al., 2000; Fletcher et al., 2001b; Longcope et al., 2005; Mandrini et al., 2006). These flare analyses have shown that $\mathrm{H} \alpha$ and UV flare ribbons are typically located at, or in the close vicinity of, the intersection of separatrices with the chromosphere. The ribbons are connected by field lines (computed from magnetic field extrapolations). These results provide evidence that flares are a consequence of localized magnetic reconnection in the corona.

Separatrices are also present when dipped field lines are tangent to the photospheric boundary. The bottom of these local magnetic dips are called "bald patches" (Titov et al., 1993). Pariat et al. (2004) found that reconnection at bald-patch separatrices is a key process during the emergence of magnetic flux through the photosphere, since this reconnection process allows the release of the dense plasma caught in the magnetic dips (it removes the plasma weight), and so it allows the magnetic field to cross the photosphere. Moreover, the computed bald patch topology let us understand the morphology of several 

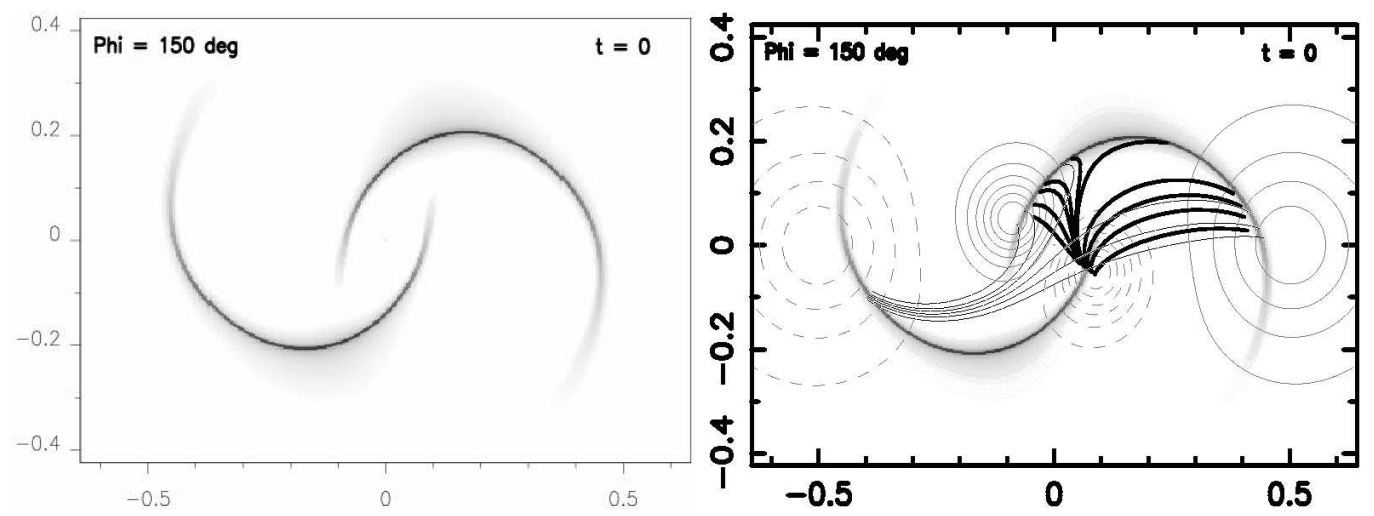

Fig. 1. Illustration of the QSLs in one of the simplest magnetic configurations (formed by two potential magnetic bipoles). The greyscale images show the distribution of the squashing degree $Q$ at the lower boundary in logarithmic scale (white: $Q=6$, black: $Q=10^{11}$ ). Two sets of field lines are shown in order to visualize the drastic change of connectivity, both sets have footpoints starting in a short segment crossing one QSL at the lower boundary. Continuous and dashed contours show positive and negative values of the vertical field component, respectively (from Aulanier et al., 2005).

small eruptive events (Aulanier et al., 1998; Mandrini et al., 2002), transition region brightenings (Fletcher et al., 2001a), and some interconnecting arcs between two ARs (Delannée and Aulanier, 1999).

In summary, there are many observed cases where the separatrices of coronal null points or bald patches let us understand the spatial localization of the energy release. However, in several ARs no such coronal null point, or bald patch, can be found and linked to the observational evidences of energy release (Démoulin et al., 1994). Of course, it is possible that the model of the coronal field is not close enough to the real field, but it was shown that a broader concept than separatrices can help us to understand the observed traces of energy release, using the same magnetic extrapolation methods, as explained below.

To address the difficulties of interpreting several flares with only separatrices, Démoulin et al. (1996a,b) proposed that "quasi-separatrix layers" (QSLs) generalize the definition of separatrices to cases where there is no coronal magnetic null and no bald patch. QSLs are regions where there is a drastic change in field line linkage (Fig. 1). The drastic change in linkage can be quantified using $Q$, called the squashing degree (computed from spatial derivatives on the magnetic connections). Then, a QSL is present at locations where $Q>>2$ (Titov et al., 2002).

Let us consider a tiny circular region in one photospheric magnetic polarity. Outside QSLs, this region is mapped following field lines to a region in the other polarity which could have a complex shape, but comparable extensions in all directions. However, if the circular region is inside a QSL, it is mapped 
to a very elongated and flatten region, ellipsoidal-like, in the other polarity. The squashing degree, $Q$, measures the aspect ratio of this ellipse; in other words, how much the initial region is squashed by the field-line mapping. $Q$ can take very high values, by many orders of magnitudes.

QSLs include, as a the limit case when $Q \rightarrow \infty$, the concept of separatrices. With separatrices the mapping changes so drastically that it is discontinuous. Let us consider a potential magnetic configuration, as in Fig. 1, formed by four magnetic concentrations at the lower boundary (e.g. photospheric level), with a normal field component, $B_{n}$, vanishing only along a line, called the inversion line. Unless the two magnetic bipoles are close to be anti-parallel, there is generally no magnetic null above the lower boundary and the magnetic connectivity is continuous, but drastically changing at the QSLs. Let us transform progressively the magnetic configuration by concentrating more and more the magnetic field in the four magnetic concentrations at the lower boundary, keeping their center of gravity at the same locations. For simplicity of the illustration, let us keep the configuration potential. As the field concentration increases, the QSLs stay basically at the same locations while they get thinner ( $Q$ increases, Démoulin et al., 1996a). When the field concentration is large enough, $Q$ becomes infinite: the QSLs becomes separatrices associated to at least one null present first at the lower boundary (and eventually in the volume). More generally, QSLs converge to separatrices in the limit where the photospheric unipolar regions are separated by flux free regions $\left(B_{n}=0\right)$. Then, analyzing QSLs permit to detect "topological" features of the magnetic field which could be related to separatrices in some limit case of the model. However, many magnetic configurations have no separatrix but they do have QSLs. The relationship between separatrices and QSLs is further analyzed in the reviews by Démoulin (2005) and Longcope (2005).

At any given region inside QSLs, two field lines, which are locally close by, diverge from each other when computed away from this region. This effect is strongest in the central part of QSLs, where the strongest mapping distortions are present (largest $Q$ values). The way field lines diverge from each other suggests that we call the central part of the QSLs a hyperbolic flux tube (HFT, Titov et al., 2002). The HFT generalizes the concept of separator: as above for QSLs, when the boundary field get more and more concentrated, the region with the highest $Q$-values converge to a null-null line (if two null points are present in the volume) or to a quasi null line (if only one null is present, Lau, 1993). However, like above with QSLs, an HFT can be present without null point in the magnetic configuration considered. The theoretical developments on QSLs and HFTs have been reviewed in Titov (2005) and Démoulin (2006).

For each studied flare, the flare ribbons were always found along, or just nearby, the intersection of QSLs with the chromosphere (e.g. Démoulin et al., 

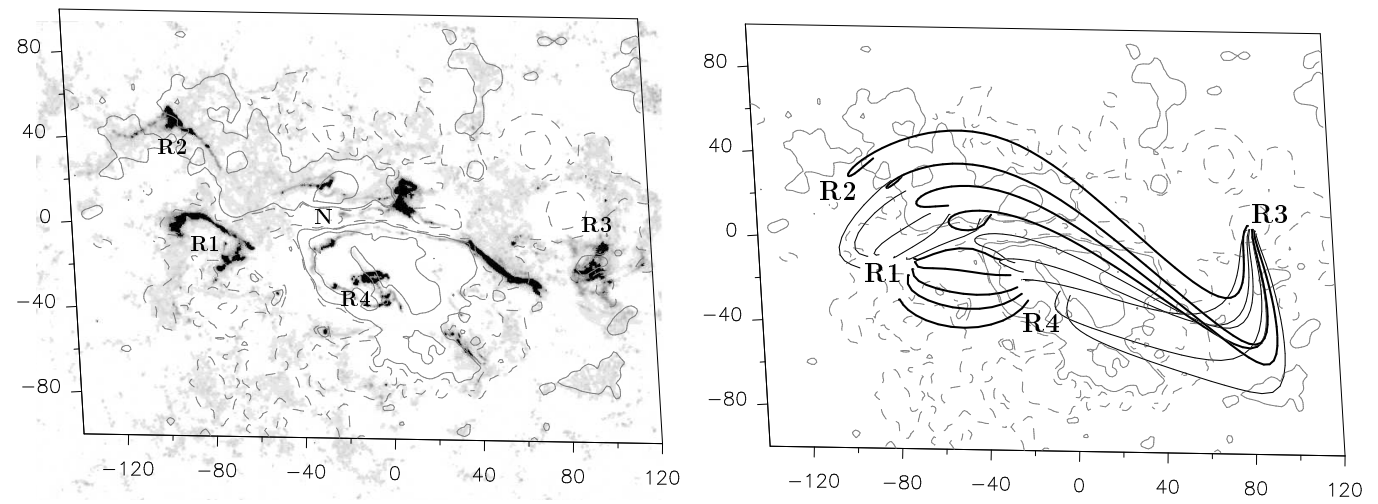

Fig. 2. Flaring processes one hour before the X17 flare on October 28, 2003. Left panel: TRACE image in $1600 \AA$ in reverse intensity scale. The main ribbons (dark regions) are labeled (R1, R2, R3, R4). The position of the only magnetic null point found in the extrapolation of the photospheric field is marked with an N. Reconnection there can only explain the brightenings seen in its close vicinity (without labels), but not the four main ribbons. Right panel: Drastic change of coronal connectivities, so QSLs, are found at or in the close vicinity of the four main ribbons. They are the result of energy release by magnetic reconnection at QSLs (notice that the null point, $\mathrm{N}$, is very low in the atmosphere, and it is not related to the X-like configuration of the field lines as seen in projection). The axes are labeled in $\mathrm{Mm}$ and the isocontours of the field correspond to \pm 100 and $\pm 1000 \mathrm{G}$ in continuous (dashed) style for the positive (negative) values (from Mandrini et al., 2006).

1997; Mandrini et al., 1997; Bagalá et al., 2000). Other examples are reviewed by Démoulin (2006). These results demonstrate that flares are coronal events, where the release of free magnetic energy is due to the presence of regions where the magnetic field line linkage changes drastically, and not necessarily discontinuously.

A flare example involving both separatrices and QSLs is given in Fig. 2. Only one magnetic null point was found in the extrapolated field; it was located at a height of only $\approx 4000 \mathrm{~km}$ above the magnetogram location (Mandrini et al., 2006). The photospheric trace of the separatrices are in the close vicinity of the brightenings observed near the null point (towards the north and south, at a distance $\leq 20 \mathrm{Mm}$, and towards the east, at a distance $\leq 40 \mathrm{Mm}$ ). Moreover, the field lines in the vicinity of the null point let us understand the shape and location of the coronal loops observed by TRACE in terms of classical reconnection at a null point. Similar coronal loops were present before and after the X17 flare showing that this localized reconnection is independent of the main flare. Before the X17 flare, four bright ribbons were also present (Fig. 2, left). They can be linked by field lines in a quadrupolar configuration (Fig. 2, right). A classical approach, considering only null points and bald patches, is not able to explain the presence of these four ribbons. However this reconnection before the X17 flare can be readily interpreted with QSLs. This is an example of a precursor that contributes to the launch of the main 
eruptive flare (X17).

\section{Formation of electric current layers}

The formation of a strong current layer in any QSL is expected with almost any kind of boundary motion across the QSL, as conjectured analytically by Démoulin et al. (1996a). The main reason is that the magnetic stress of very distant regions, generated by plasma flows, can be brought close to one another, typically over the QSL thickness (the magnetic stress is mainly transported by Alfvén waves which are damped later in the quasi-equilibrium state).

MHD numerical simulations are required to analyze the evolution of general magnetic configurations having QSLs. A numerical difficulty is that the currents are expected to form on the scale of the QSL thickness, a scale that can be many orders of magnitude lower than the global scale of the studied magnetic configuration. In a simulation the thinnest scales are limited by the size of the numerical cells. All the current layers should be broader; this is attained by selecting a resistivity large enough. The MHD simulations of Longcope and Strauss (1994) and Milano et al. (1999) showed that currents did form along QSLs. In particular, Longcope and Strauss showed that the thickness of the current layers decreases exponentially with time; this implies an exponential growth of the electric current density.

It is worth noting that QSLs were not present in the initial field configurations of the above simulations. They were dynamically formed by the prescribed boundary motions, which consisted of vortices with stagnation points in between them. The numerical simulations of Galsgaard et al. (2003) were aimed to address the formation of current layers in pre-existing QSLs. Unfortunately, they considered very broad initial QSLs, with a thickness of about one tenth of the numerical domain, so only weak currents were formed. The selected boundary motions produced a stagnation point inside the numerical domain, which resulted in the formation of a strong current layer, as predicted analytically by Titov et al. (2003). So, in fact, Galsgaard et al. (2003) studied the build up of currents associated to other much thinner QSLs which are formed dynamically by the boundary motions. In this way, their results extended those of Longcope and Strauss (1994) and Milano et al. (1999).

When thin QSLs are present in the initial configurations, the MHD simulations of Aulanier et al. (2005) did show that narrow current layers developed in general at the QSLs, whatever the footpoint motions are and even for relatively small displacements (Fig. 3). Of course, the precise current distribution in the QSLs depends on the type of motions imposed, but the strongest electric 

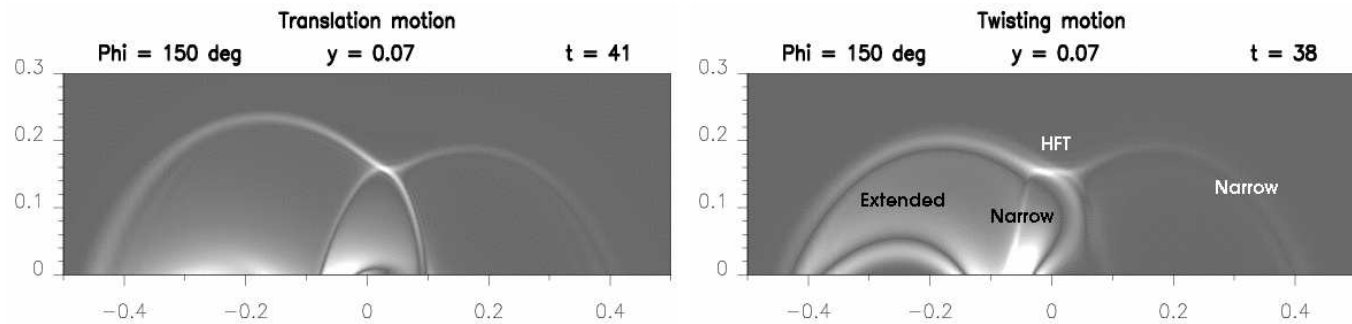

Fig. 3. Formation of strong electric currents at QSLs after the configuration shown in Fig. 1 has been evolved by an MHD simulation. The figures are vertical cuts of the 3 -D configuration (this cut would correspond to a horizontal line at $y=0.07$ in Fig. 1). The plasma flow implemented at the lower boundary corresponds to a global translation (resp. twisting motion) of the smallest positive magnetic polarity (located at $\approx-0.09,0.05)$. The current intensity scales linearly with the grey-scale intensity, white being the highest value. Each image shows the co-existence of extended currents, in a large part of the simulation volume, and narrow currents layers within the whole QSLs (from Aulanier et al., 2005).
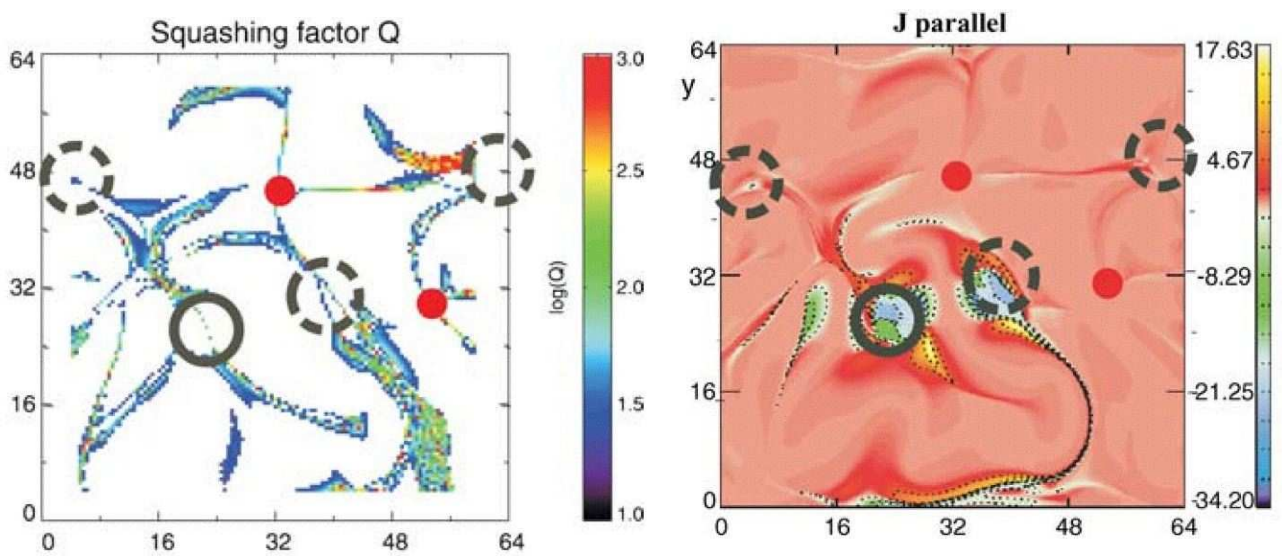

Fig. 4. QSLs and electric current layers in a magnetic configuration computed from a magnetogram. Left panel: The images show the distribution of the squashing degree $Q$ at the lower boundary in logarithmic scale. Continuous and dashed circles show the approximate size and location of the main positive and negative magnetic flux concentrations. The two dots show the positions of two chromospheric magnetic nulls. Right panel: Corresponding electric current patterns created by an MHD evolution using observed flows applied to the extrapolated field. The grey levels represent the current parallel to the magnetic field integrated along the magnetic field lines in the volume (from Büchner, 2006).

currents develop at (or nearby) the HFT, so where the QSLs are the thinnest. This current layer becomes thinner and stronger with time; then, significant reconnection occurs when the currents reach the dissipative scale.

Büchner (2006) analyzed a EUV bright point well-observed by TRACE. He extrapolated the observed photospheric field and introduced a stratified atmosphere. The magnetic topology of the configuration is complex since two null points, their associated separatrices, as well as QSLs are present (Fig. 4, left). 


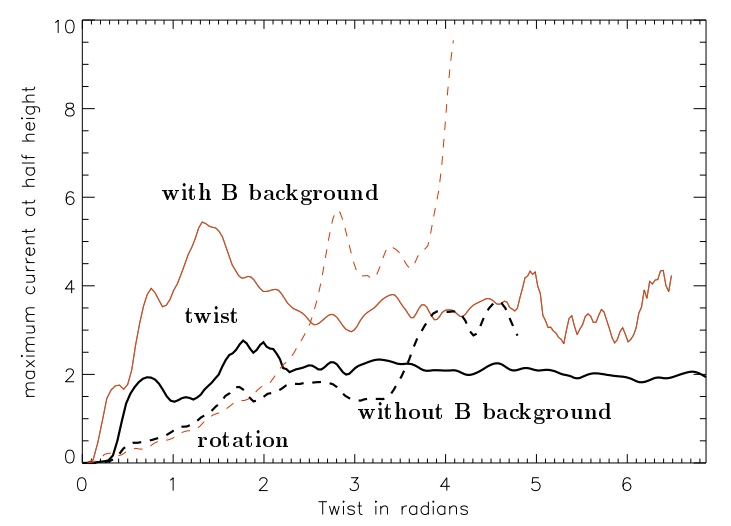

Fig. 5. Maximum electric current found in MHD simulations as function of the twist/rotation imposed by boundary flows. The initial configuration is formed by two parallel and potential flux tubes. The solid lines correspond to twisting motions imposed in each magnetic polarity and dashed lines to relative rotation motions of both polarities (correspond to an imposed orbital-like motion of the polarities). The thin grey (or brown/red for the color version) lines correspond to a case with an added uniform background field, making the QSLs thicker than in the two previous cases (black or thick lines). The current build up is stronger with thicker QSLs for both cases (twisting and rotation motions, thin lines). There is as well more energy released during reconnection with thicker QSLs (from de Moortel and Galsgaard, 2006b).

This initial configuration was evolved applying the observed photospheric motions. Strong electric currents appeared mostly at the QSLs (Fig. 4, right). The current pattern follows mostly the observed EUV brightenings showing that the strongest current densities are indeed associated to the strongest magnetic energy dissipation. Moreover, the currents are dominantly aligned with the magnetic field, but the significant $\beta$ considered in the simulation also introduces a current component perpendicular to the magnetic field. This perpendicular component is also strongly peaked at the QSLs. Finally, it is worth noting that weaker currents are formed on the separatrices because the observed flows are stronger in the QSL regions, and also plausibly because the thinner current layers, formed at separatrices, are dissipated faster than those formed at QSLs (see next section).

\section{Magnetic reconnection}

Most of the studies of magnetic reconnection have been done in $2-\mathrm{D}$ and $2 \frac{1}{2}$-D configurations (the magnetic and velocity fields have three components which depend only on two spatial coordinates). These studies have set the reconnection process on physical ground (see, e.g., the review of Priest and Forbes, 2000). Reconnection in $2-\mathrm{D}$ and $2 \frac{1}{2}$-D can be defined in many differrent, but equivalent, ways. However, it is not obvious which of these definitions, if any, 
can be generalized to 3-D configurations (Démoulin et al., 1996a; Hesse et al., 2005, and references therein). For example, 2-D and $2 \frac{1}{2}$-D reconnection can be defined by the presence of plasma flows across the magnetic separatrices. This generalizes to 3-D only when null points or bald patches are present.

Hesse and Schindler (1988) and Schindler et al. (1988) argued that, in many non-particular configurations, the separatrices are structurally unstable features (they disappear) when perturbing slightly a $2 \frac{1}{2}$-D configuration to form a $3-\mathrm{D}$ one (so breaking the spatial invariance in one direction). An example is a twisted flux tube embedded in an arcade. As soon as the configuration is no longer invariant along the flux tube, the separatrices disappear, and we can pass continously from field lines inside to outside the twisted flux tube as in a magnetic arcade. In their view, 3-D reconnection can occur at any location, provided the magnetic dissipation is locally enhanced. This contrasts sharply with most of the previous studies on 2-D and $2 \frac{1}{2}$-D reconnection! Using Euler potentials, they developed a general framework for 3-D reconnection based on localized nonideal regions, where a finite component of the electric field is present along the magnetic field. They propose the presence of this parallel electric component as a general definition of 3-D reconnection.

However, the above structural instability is no longer present when the notion of separatrices is generalized to QSLs (Démoulin et al., 1996b). In the examples of Hesse and Schindler (1988) and Schindler et al. (1988) the discontinuous field-line linkage in $2 \frac{1}{2}$-D configurations is simply replaced by a drastic change of the field-line linkage in 3-D. This drastic change could happen on spatial scales of the order or below $1 \mathrm{~m}$, so scales where MHD is indeed not applicable, if the magnetic twist is of few turns (Démoulin et al., 1996b). Since strong current layers are formed at the QSLs with almost any evolution of the field (Section 3), an electric field component along the magnetic field is naturally present at QSLs. This approach indeed complements the theory of Hesse and Schindler by localizing the non-ideal regions, that is to say, the regions where reconnection can occur. In agreement with Hesse and Schindler, it recognizes that transforming the magnetic connectivities is the main property of 3-D reconnection. This property is indeed a general definition of reconnection (in 2-D as in 3-D, with separatrices and with QSLs). The QSL approach permits also to link this 3 -D reconnection to previous 2 -D and $2 \frac{1}{2}$-D reconnection studies, generalising the concept of separatrices.

Some of the characteristics found previously in studies of $2-\mathrm{D}$ and $2 \frac{1}{2}$-D reconnection are obviously present in 3-D reconnection (Titov et al., 2003; Galsgaard et al., 2003; de Moortel and Galsgaard, 2006a; Pontin et al., 2005; Aulanier et al., 2006). For example, the intensity of the current layer and of the reconnection rate are important at the HFT (the generalization of the separator). Outflow jets are also present. The outflow velocity is large, compared to the inflow velocity, but not necessarily super-Alfvénic. There are also im- 


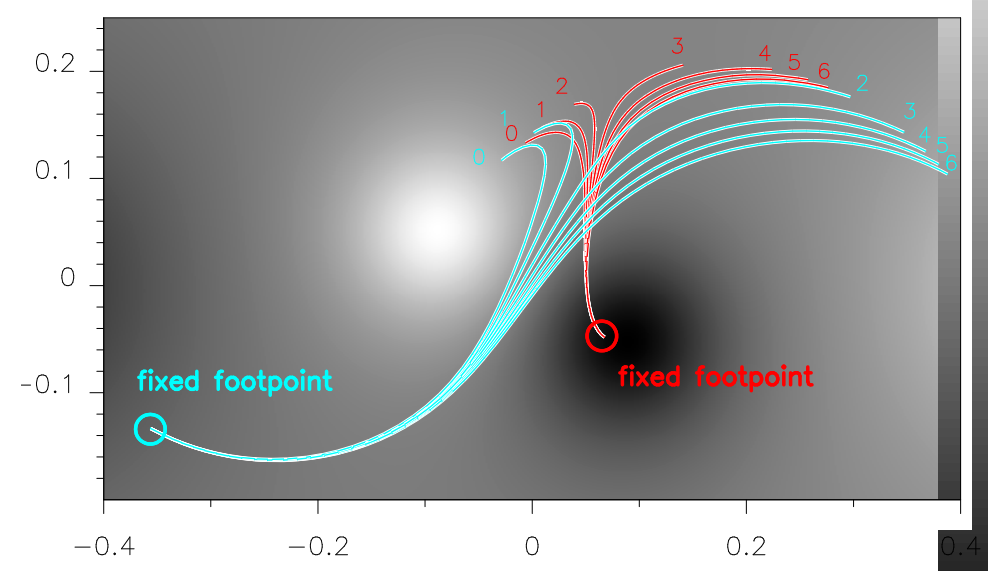

Fig. 6. Magnetic reconnection occurring in the configuration of Figure 1 after it was evolved by a twisting motion (located in the smallest positive magnetic polarity). The evolution of two slip-running field lines with fixed footpoints in the negative polarities (for convenience of representation) are represented with a fixed time step. As reconnection proceeds, each field line progressively changes its connection, shifting along the QSL. The shift per unit time is maximum when the field line is in the centre of the QSL, where the squashing degree $Q$ has the highest values (so at the HFT). This motion of the connectivity along the QSL is accompanied by much smaller motions across the QSL (not shown) which are related to the usual displacement of chromospheric ribbons (from Aulanier et al., 2006).

portant new features of 3-D reconnection, as discussed below They are present in such a peculiar limit form in $2-\mathrm{D}$ and $2 \frac{1}{2}$-D configurations that they were overlooked.

A first new feature is the amount of energy released in function of the initial QSL thickness. During any evolution strong current layers build up at the QSLs, and they get narrower with time (Section 3). For initially broader QSLs, the time required for the current layer to be pinched down to the dissipative scales is longer. This implies that both the free-energy stored before reconnection and the amount of reconnected flux are more important with broader initial QSLs (Aulanier et al., 2005). This has been recently confirmed by de Moortel and Galsgaard (2006b, Fig. 5). This result contrasts with the long history of the separatrix-related flare models which invoke the formation of long current sheets during the energy build-up phase. With the above new results, these separatrix-models are more relevant to coronal heating (since they are expected to have a low amount of free magnetic energy stored in current layers and frequent episodes of energy release).

Another new feature is the development of a fast slippage of magnetic field lines along QSLs. This was envisioned by Priest and Forbes (1992), then the concept was elaborated by Priest and Démoulin (1995). They suppose a stationary evolution (i.e. the magnetic field at a given point stays unchanged, and 

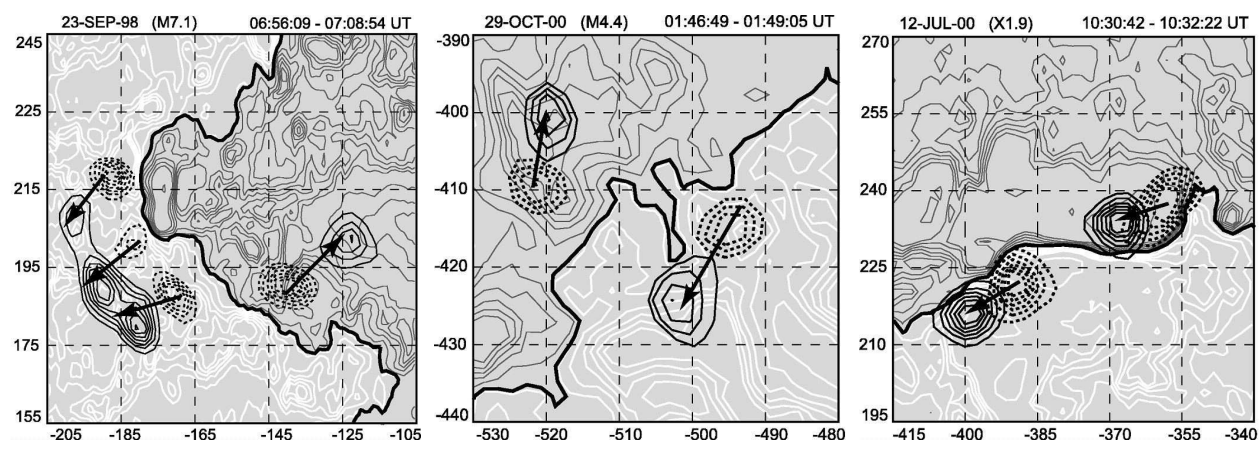

Fig. 7. Example of the three main types of X-ray source motions as observed with the Hard X-Ray Telescope aboard Yohkoh satellite. Left: Motions away from the photospheric inversion line (PIL). Centre: Antiparallel motions along the PIL. Right: Motions in the same direction along the PIL (from Bogachev et al., 2005).

there is a stationary flow which transports the magnetic flux tubes). Moreover, they also suppose that the magnetic field stays a potential field (i.e. with negligible electric currents). They proposed that reconnection occurs in a QSL when the sub-Alfvénic displacement of one field line footpoint across the QSL results in a super-Alfvénic displacement of the other footpoint. However, this approach does not take into account the line tying of both field line footpoints at the boundary ( $\approx$ photosphere), nor the evolution of the electric currents. This limitation was removed analytically by a kinematic model (Priest et al., 2003) and by MHD numerical simulations (Pontin et al., 2005; DeVore et al., 2005). The next step was to consider thin enough initial QSLs. For sufficiently thin QSLs, field line footpoints can shift super-Alfvénically along the QSLs (Aulanier et al., 2006, Fig. 6). This process is called slip-running reconnection, in opposition to mild and slow diffusive slippage which occurs for low values of the squashing degree.

Chromospheric flare ribbons are observed in $\mathrm{H} \alpha$ and in EUV to separate from each another on both sides of the photospheric inversion line (PIL) of the magnetic field (e.g. Svestka and Cliver, 1992; Asai et al., 2004). The flare loops have a shear which usually decreases with time (e.g. Asai et al., 2003; Su et al., 2006). This is interpreted as magnetic reconnection progressing from inside the strongly sheared core field towards a more potential field away from the PIL. Recent analysis of Yohkoh/HXT (Hard X-ray Telescope) and RHESSI (Ramaty High Energy Solar Spectroscopic Imager) observations have also revealed complex motions of hard X-ray (HXR) compact sources (Fletcher and Hudson, 2002; Krucker et al., 2003). Bogachev et al. (2005) classified these motions in three main types (Fig. 7). The first type, with motions away of the PIL, corresponds to the usual separation of flare ribbons; it is one main consequence of reconnection in standard 2-D models of eruptive flares. The second type, with anti-parrallel motions along the PIL, is not a logical consequence of 2-D and $2 \frac{1}{2}$-D reconnection, nor of $3-\mathrm{D}$ reconnection with separatrices. It is usu- 
ally interpreted as successive reconnections in a configuration with a spatial gradient of magnetic shear. Finally, the third type, with comparable motions along the PIL, is interpreted as successive reconnections "propagating" along the magnetic arcade (Grigis and Benz, 2005).

The 3-D reconnection at QSLs can explain the three types of observed ribbon motions just like classical 3-D reconnection with separatrices. Furthermore, the slip-running reconnection offers a natural explanation for the anti-parrallel motions of HXR sources. Particles accelerated by a super-Dreicer electric field at slightly different times will follow the changing magnetic connectivities as slip-running reconnection proceeds. Such particles impact regions in the low atmosphere that will naturally drift along the flare ribbons. The motions along the ribbons will depend not only upon the thickness of the QSL, the reconnection rate, but also upon the relative position of the acceleration site with respect to the HFT in the corona. If the coronal acceleration sites slightly change position with time, this change will be amplified by the field line mapping at the footpoints and produce rapid change along the ribbons. So the HXR sources are expected to have important and complex motions, with an expected average drift along the flare ribbons. Here we reach one of the next challenges: coupling MHD simulations with models of particle acceleration.

\section{Challenges}

There are still several challenges to overcome before understanding the full physics of solar flares. Here we consider those which are related to the issue of flare energetics.

The results reported in Section 2 were obtained with confined flares. Their magnetic configurations are significantly quadrupolar and/or the magnetic shear is low enough so that the QSL location is dominantly defined by the presence of strong flux concentrations at the photospheric level. In such cases the QSL locations are simply shifted with an increasing magnetic shear, and linear force-free field extrapolations of the photospheric magnetograms are usually sufficient to find the QSLs involved in the observed energy release. However, new QSLs appear when the magnetic twist is important (typically more than about one turn). These QSLs become exponentially thinner as the twist increases, and their locations are dominantly defined by the electric current distributions. The chromospheric footprints of these QSLs have characteristic J-shapes organized according to the sign of the magnetic twist (Démoulin et al., 1996b). Examples of such J-shaped ribbons have been reported in prominence eruptions and eruptive flares (e.g. Martin and Harvey, 1979; Moore et al., 1995; Pevtsov et al., 1996; Williams et al., 2005). It is a challenge to model such highly stressed magnetic configurations. For example, 
for the flare events on October 28, 2003, it was only meaningful to compute the large-scale (so less sheared) quadrupolar connectivity before the main flare (Fig. 2).

Non-linear force free field extrapolations of strongly sheared, and even twisted, configurations of eruptive flares are difficult to derive from photospheric magnetograms. First, the transverse field is only available in the strong field regions (typically $>200 \mathrm{G}$ ), then the $180^{\circ}$ degree ambiguity should be solved and the extrapolations require the electric currents which are computed using the spatial derivative of noisy data (so enhancing the noise over the signal). Significant advances are expected with THEMIS and Hinode vector magnetograms. Next, computing the coronal magnetic field from photospheric data is a much more difficult problem than can be appreciated from the apparently simple form of the governing equations. It is a non-linear, non local, and not necessarily well posed problem (depending on the boundary condition used). It is more difficult than direct MHD simulations, since observed data give boundary conditions at a given time without any information on the temporal evolution. Only recently has significant progress been made for highly sheared configurations (e.g. Valori et al., 2005; Inhester and Wiegelmann, 2006). Different methods have been evaluated in blind tests builded with analytical force-free field models having an arcade-like topology (Schrijver et al., 2006). Some methods have been tested with a relative success on a twisted force-free field configuration comparable to what is expected in eruptive flares (Wiegelmann et al., 2006). These progresses are encouraging even if there is still a long way before routine applications of non-linear force-free extrapolation methods to vector magnetograms.

Running an MHD simulation is another approach to model the magnetic configurations. This requires as boundary conditions an "initial" magnetogram of the vertical component and the time evolution of the plasma velocity from the "initial" to the "observed" time at the photospheric level. The "initial" coronal field needs to be derived from an extrapolation (which could be potential if the "initial" time is sufficiently far from the "observed" one, so that the initial conditions are mostly washed out). A standard method to derive the photospheric velocities is the local correlation tracking (LCT). Chae (2001) first applied it to magnetograms. Improved methods, taking into account the induction equation, have been developed (e.g. Kusano et al., 2004; Welsch et al., 2004; Schuck, 2006). The challenge is to accurately determine the flow pattern during typically a week. On the MHD simulation side, the challenge is to be able to describe accurately all the magnetic evolution; in particular, the formation, then reconnection, of thin current layers to finally end up with a field configuration comparable with the observed one.

Current layers often behave like monolithic structures in the 3D MHD simulations described Section 4, probably because only a limited range of spatial 


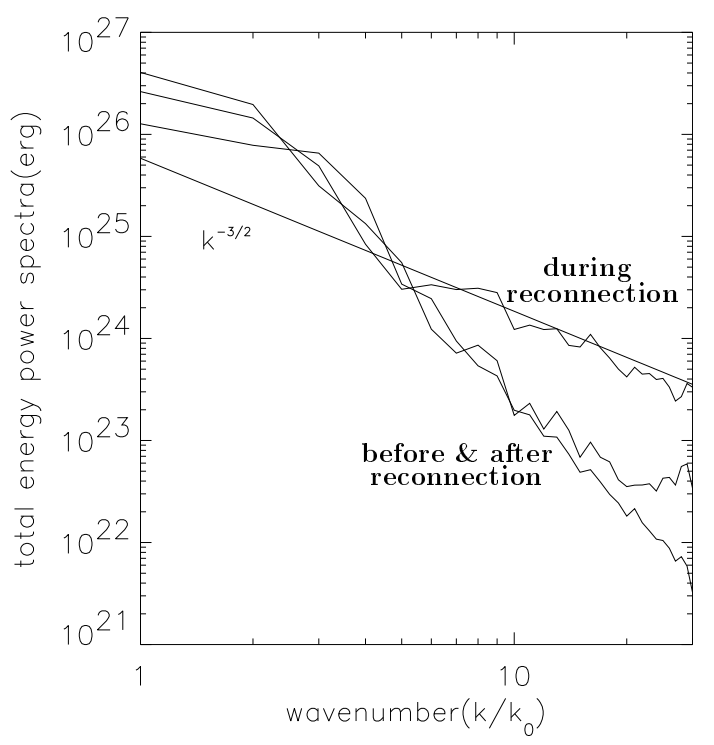

Fig. 8. Energy power spectra before, during, and after the reconnection between two twisted flux tubes. The spectrum is flatter during the reconnection and the $3 / 2$ slope (straight line) corresponds to the Kraichnan regime (Kraichnan, 1965) of fully developed turbulence (from Milano et al., 1999).

scales are treated. The resistivity is adapted to damp the spatial modes at scales comparable to the size of the numerical cells in order to assure numerical stability. However, in the $2 \frac{1}{2}$-D MHD simulations of Karpen et al. (1990), evidence is found that magnetic reconnection of an extended current sheet induces a current filamentation in the post-reconnected region. Shibata and Tanuma (2001) proposed that any extended current layer becomes unstable to the tearing mode; and that, during the nonlinear evolution, secondary tearing occurs at successively lower scales breaking the reconnecting region into smaller and smaller current sheets. This process creates a series of twisted flux tubes that become thinner as reconnection occurs at smaller scales. The formation of twisted flux tubes has been found by Archontis et al. (2006), in a 3-D MHD simulation, at the interface between an emerging flux tube and a horizontal field. In the different context of magnetic turbulence, this development of fine structures was proposed by Larosa and Moore (1993). Reduced MHD simulations of Hendrix and van Hoven (1996) and Milano et al. (1999) support this cascade of energy to small scales. For example, the development of a Kraichnan's energy spectra is obtained during the peak of the reconnection rate (Fig. 8). This turbulence is expected to break any monolithic current layer into many smaller current layers, so that magnetic reconnection, as well as particle acceleration, will occur at many locations (which are still very close at the scale of the flaring region). Such results need to be extended to a larger range of spatial scales, so larger hydrodynamic and magnetic Reynolds numbers to better represent the processes occurring in the corona.

As smaller scales become involved in the reconnection process, it becomes 
necessary to consider a more complete set of physical equations. In particular Ohm's law needs to be better described than with a uniform resistivity. In particular the Hall term is expected to be significant (e.g. Ma and Bhattacharjee, 2001; Morales et al., 2005). Moreover, microscopic plasma instabilities are expected to develop, and these may lead to the development of an anomalous resistivity. Moreover, the electric fields generated exceed the Dreicer electric field by several orders of magnitude. At this point, MHD will not be able to describe the local processes involved in the reconnection of the thin layers, and kinetic theory is required (see e.g. the review of Heyvaerts, 2000). However, describing the full configuration by kinetic theory is not possible even with the super-computers that will become available in the near future. The logical strategy is to use kinetic theory only in the thin volumes where MHD breaks down, i.e. in the reconnecting current layers (Büchner, 2007). This approach is needed in order to link the global physics described by MHD (Section 4) to the local acceleration of particles in multiple accelerating regions (e.g. Onofri et al., 2006; Turkmani et al., 2006; Dauphin et al., 2007).

MHD, and in particular magnetic extrapolations and the physics involved in QSLs, are needed to understand where electric current layers are forming; and then, where reconnection is occurring. This approach is well adapted to understand the temporal evolution of flares, as observed with imaging data (like Yohkoh, SOHO, THEMIS, TRACE, Hinode) since it allows us to localize the reconnection region within the global magnetic configuration. MHD simulations involving separatrices, and their generalization, QSLs, are expected to be able, in the near future, to describe the evolution of flare ribbons; which in turn will provide a global view of the reconnection process in flares. However, MHD cannot describe the intimate physics of the reconnection processes, nor particle acceleration, since these processes need to be described by kinetic theory. Coupling these approaches is expected to give a global understanding of the flare/CME physics.

Acknowledgments: I thank G. Aulanier, C.H. Mandrini, and L. van DrielGesztelyi and the two referees for their help in improving the manuscript.

\section{References}

Aly, J. J., 1990. Do Current Sheets Necessarily Form in 3D Sheared Magnetic Force-Free Fields ? In: The Dynamic Sun. p. 176.

Archontis, V., Galsgaard, K., Moreno-Insertis, F., Hood, A. W., 2006. Threedimensional Plasmoid Evolution in the Solar Atmosphere. ApJ, 645, L161L164.

Asai, A., Ishii, T. T., Kurokawa, H., Yokoyama, T., Shimojo, M., 2003. Evolution of Conjugate Footpoints inside Flare Ribbons during a Great TwoRibbon Flare on 2001 April 10. ApJ, 586, 624-629. 
Asai, A., Yokoyama, T., Shimojo, M., Masuda, S., Kurokawa, H., Shibata, K., 2004. Flare Ribbon Expansion and Energy Release Rate. ApJ, 611, 557-567.

Aulanier, G., DeLuca, E. E., Antiochos, S. K., McMullen, R. A., Golub, L., 2000. The Topology and Evolution of the Bastille Day Flare. ApJ, 540, $1126-1142$.

Aulanier, G., Démoulin, P., Schmieder, B., Fang, C., Tang, Y. H., 1998. Magnetohydrostatic Model of a Bald-Patch Flare. Solar Physics, 183, 369-388.

Aulanier, G., Pariat, E., Démoulin, P., 2005. Current sheet formation in quasiseparatrix layers and hyperbolic flux tubes. A\&A, 444, 961-976.

Aulanier, G., Pariat, E., Démoulin, P., Devore, C. R., 2006. Slip-Running Reconnection in Quasi-Separatrix Layers. Solar Physics, 238, 347-376.

Bagalá, L. G., Mandrini, C. H., Rovira, M. G., Démoulin, P., 2000. Magnetic reconnection: a common origin for flares and $\mathrm{AR}$ interconnecting arcs. A\&A, 363, 779-788.

Bogachev, S. A., Somov, B. V., Kosugi, T., Sakao, T., 2005. The Motions of the Hard X-Ray Sources in Solar Flares: Images and Statistics. ApJ, 630, $561-572$.

Büchner, J., 2006. Locating Current Sheets in the Solar Corona. Space Science Reviews 122, 149-160.

Büchner, J., 2007. Simulation of the electric fields created by magnetic reconnection in the solar corona by combining large scale and plasma kinetic approaches. Advances in Space Research, submitted.

Chae, J., 2001. Observational Determination of the Rate of Magnetic Helicity Transport through the Solar Surface via the Horizontal Motion of Field Line Footpoints. ApJ, 560, L95-L98.

Dauphin, C., Vilmer, N., Anastasiadis, A., 2007. Particle acceleration and radiation in a flaring complex solar active regions modelled by cellular automata. A\&A, , submitted.

de Moortel, I., Galsgaard, K., 2006a. Numerical modelling of 3D reconnection due to rotational footpoint motions. A\&A, 451, 1101-1115.

de Moortel, I., Galsgaard, K., 2006b. Numerical modelling of 3D reconnection. II. Comparison between rotational and spinning footpoint motions. A\&A, 459, 627-639.

Delannée, C., Aulanier, G., 1999. CME Associated with Transequatorial Loops and a Bald Patch Flare. Solar Physics, 190, 107-129.

Démoulin, P., 2005. Magnetic Topologies: where Will Reconnection Occur ? In: Innes, D. E., Lagg, A., Solanki, S. A. (Eds.), ESA SP-596: Chromospheric and Coronal Magnetic Fields.

Démoulin, P., 2006. Extending the concept of separatrices to QSLs for magnetic reconnection. Advances in Space Research 37, 1269-1282.

Démoulin, P., Bagalá, L. G., Mandrini, C. H., Hénoux, J. C., Rovira, M. G., 1997. Quasi-separatrix layers in solar flares. II. Observed magnetic configurations. A\&A, 325, 305-317.

Démoulin, P., Hénoux, J. C., Priest, E. R., Mandrini, C. H., 1996a. QuasiSeparatrix layers in solar flares. I. Method. A\&A, 308, 643-655. 
Démoulin, P., Mandrini, C. H., Rovira, M. G., Hénoux, J. C., Machado, M. E., 1994. Interpretation of multiwavelength observations of November 5, 1980 solar flares by the magnetic topology of AR 2766. Solar Physics, 150, 221243.

Démoulin, P., Priest, E. R., Lonie, D. P., 1996b. Three-dimensional magnetic reconnection without null points 2. Application to twisted flux tubes. JGR, 101(A10), 7631-7646.

DeVore, C. R., Antiochos, S. K., Aulanier, G., 2005. Solar Prominence Interactions. ApJ, 629, 1122-1134.

Fletcher, L., Hudson, H. S., 2002. Spectral and Spatial Variations of Flare Hard X-ray Footpoints. Solar Physics, 210, 307-321.

Fletcher, L., López Fuentes, M. C., Mandrini, C. H., Schmieder, B., Démoulin, P., Mason, H. E., Young, P. R., Nitta, N., 2001a. A Relationship Between Transition Region Brightenings, Abundances, and Magnetic Topology. Solar Physics, 203, 255-287.

Fletcher, L., Metcalf, T. R., Alexander, D., Brown, D. S., Ryder, L. A., 2001b. Evidence for the Flare Trigger Site and Three-Dimensional Reconnection in Multiwavelength Observations of a Solar Flare. ApJ, 554, 451-463.

Gaizauskas, V., Mandrini, C. H., Démoulin, P., Luoni, M. L., Rovira, M. G., 1998. Interactions between nested sunspots. II. A confined X1 flare in a delta-type sunspot. A\&A, 332, 353-366.

Galsgaard, K., Titov, V. S., Neukirch, T., 2003. Magnetic Pinching of Hyperbolic Flux Tubes. II. Dynamic Numerical Model. ApJ, 595, 506-516.

Gorbachev, V. S., Somov, B. V., 1988. Photospheric vortex flows as a cause for two-ribbon flares - A topological model. Solar Physics, 117, 77-88.

Grigis, P. C., Benz, A. O., 2005. The Evolution of Reconnection along an Arcade of Magnetic Loops. ApJ, 625, L143-L146.

Hendrix, D. L., van Hoven, G., 1996. Magnetohydrodynamic Turbulence and Implications for Solar Coronal Heating. ApJ, 467, 887-893.

Hesse, M., Forbes, T. G., Birn, J., 2005. On the Relation between Reconnected Magnetic Flux and Parallel Electric Fields in the Solar Corona. ApJ, 631, $1227-1238$.

Hesse, M., Schindler, K., 1988. A theoretical foundation of general magnetic reconnection. JGR, 93(A12), 5559-5567.

Heyvaerts, J., 2000. Introduction to MHD. In: Rozelot, J. P., Klein, L., Vial, J.-C. (Eds.), LNP Vol. 553: Transport and Energy Conversion in the Heliosphere. pp. 1-60.

Inhester, B., Wiegelmann, T., 2006. Nonlinear Force-Free Magnetic Field Extrapolations: Comparison of the Grad Rubin and Wheatland Sturrock Roumeliotis Algorithm. Solar Physics, 235, 201-221.

Karpen, J. T., Antiochos, S. K., Devore, C. R., 1990. On the formation of current sheets in the solar corona. ApJ, 356, L67-L70.

Kraichnan, R. H., 1965. Inertial-Range Spectrum of Hydromagnetic Turbulence. Physics of fluid 8, 1385-1387.

Krucker, S., Hurford, G. J., Lin, R. P., 2003. Hard X-Ray Source Motions in 
the 2002 July 23 Gamma-Ray Flare. ApJ, 595, L103-L106.

Kusano, K., Maeshiro, T., Yokoyama, T., Sakurai, T., 2004. Study of Magnetic Helicity in the Solar Corona. In: Sakurai, T., Sekii, T. (Eds.), ASP Conf. Ser. 325: The Solar-B Mission and the Forefront of Solar Physics. pp. 175-184.

Larosa, T. N., Moore, R. L., 1993. A Mechanism for Bulk Energization in the Impulsive Phase of Solar Flares: MHD Turbulent Cascade. ApJ, 418, 912-918.

Lau, Y. T., 1993. Magnetic Nulls and Topology in a Class of Solar Flare Models. Solar Physics, 148, 301-324.

Lau, Y.-T., Finn, J. M., 1990. Three-dimensional kinematic reconnection in the presence of field nulls and closed field lines. ApJ, 350, 672-691.

Longcope, D. W., 2005. Topological Methods for the Analysis of Solar Magnetic Fields. Living Reviews in Solar Physics 2, 1-58.

Longcope, D. W., Cowley, S. C., 1996. Current sheet formation along threedimensional magnetic separators. Physics of Plasmas 3, 2885-2897.

Longcope, D. W., Klapper, I., 2002. A General Theory of Connectivity and Current Sheets in Coronal Magnetic Fields Anchored to Discrete Sources. ApJ, 579, 468-481.

Longcope, D. W., McKenzie, D., Cirtain, J., Scott , J., 2005. Observations of Separator Reconnection to an Emerging Active Region. ApJ, 630, 596-614.

Longcope, D. W., Silva, A. V. R., 1998. A current ribbon model for energy storage and release with application to the flare of 7 January 1992. Solar Physics, 179, 349-377.

Longcope, D. W., Strauss, H. R., 1994. The form of ideal current layers in line-tied magnetic fields. ApJ, 437, 851-859.

Low, B. C., Wolfson, R., 1988. Spontaneous formation of electric current sheets and the origin of solar flares. ApJ, 324, 574-581.

Ma, Z. W., Bhattacharjee, A., 2001. Hall magnetohydrodynamic reconnection: The Geospace Environment Modeling challenge. JGR, 106, 3773-3782.

Mandrini, C. H., Démoulin, P., Bagalá, L. G., van Driel-Gesztelyi, L., Hénoux, J. C., Schmieder, B., Rovira, M. G., 1997. Evidence of Magnetic Reconnection from $\mathrm{H} \alpha$, Soft X-Ray and Photospheric Magnetic Field Observations. Solar Physics, 174, 229-240.

Mandrini, C. H., Demoulin, P., Schmieder, B., Deluca, E. E., Pariat, E., Uddin, W., 2006. Companion Event and Precursor of the X17 Flare on 28 October 2003. Solar Physics, 238, 293-312.

Mandrini, C. H., Démoulin, P., Schmieder, B., Deng, Y. Y., Rudawy, P., 2002. The role of magnetic bald patches in surges and arch filament systems. A\&A, 391, 317-329.

Mandrini, C. H., Rovira, M. G., Démoulin, P., Hénoux, J. C., Machado, M. E., Wilkinson, L. K., 1993. Evidence for magnetic reconnection in large-scale magnetic structures in solar flares. A\&A, 272, 609-620.

Martin, S. F., Harvey, K. H., 1979. Ephemeral active regions during solar minimum. Solar Physics, 64, 93-108.

Milano, L. J., Dmitruk, P., Mandrini, C. H., Gómez, D. O., Démoulin, P., 
1999. Quasi-Separatrix Layers in a Reduced Magnetohydrodynamic Model of a Coronal Loop. ApJ, 521, 889-897.

Molodenskii, M. M., Syrovatskii, S. I., 1977. Magnetic fields of active regions and their zero points. Soviet Astronomy 21, 734-741.

Moore, R. L., Larosa, T. N., Orwig, L. E., 1995. The wall of reconnectiondriven magnetohydrodynamic turbulence in a large solar flare. ApJ, 438, 985-966.

Morales, L. F., Dasso, S., Gómez, D. O., 2005. Hall effect in incompressible magnetic reconnection. JGR, 110, 4204.

Onofri, M., Isliker, H., Vlahos, L., 2006. Stochastic Acceleration in Turbulent Electric Fields Generated by 3D Reconnection. Physical Review Letters 96 (15), 151102-151105.

Pariat, E., Aulanier, G., Schmieder, B., Georgoulis, M. K., Rust, D. M., Bernasconi, P. N., 2004. Resistive Emergence of Undulatory Flux Tubes. ApJ, 614, 1099-1112.

Pevtsov, A. A., Canfield, R. C., Zirin, H., 1996. Reconnection and Helicity in a Solar Flare. ApJ, 473, 533-538.

Pontin, D. I., Galsgaard, K., Hornig, G., Priest, E. R., 2005. A fully magnetohydrodynamic simulation of 3D non-null reconnection. Phys. Plasmas 12, 052307-10.

Priest, E., Forbes, T., 2000. Magnetic reconnection : MHD theory and applications. Cambridge University Press, Cambridge, UK.

Priest, E. R., Démoulin, P., 1995. Three-dimensional magnetic reconnection without null points. 1. Basic theory of magnetic flipping. JGR, 100(A9), 23443-23464.

Priest, E. R., Forbes, T. G., 1992. Magnetic flipping - Reconnection in three dimensions without null points. JGR, 97, 1521-1531.

Priest, E. R., Hornig, G., Pontin, D. I., 2003. On the nature of threedimensional magnetic reconnection. JGR, 108(A7), 1285.

Schindler, K., Hesse, M., Birn, J., 1988. General magnetic reconnection, parallel electric fields, and helicity. JGR, 93(A12), 5547-5557.

Schrijver, C. J., Derosa, M. L., Metcalf, T. R., Liu, Y., McTiernan, J., Régnier, S., Valori, G., Wheatland, M. S., Wiegelmann, T., 2006. Nonlinear ForceFree Modeling of Coronal Magnetic Fields Part I: A Quantitative Comparison of Methods. Solar Physics, 235, 161-190.

Schuck, P. W., 2006. Tracking Magnetic Footpoints with the Magnetic Induction Equation. ApJ, 646, 1358-1391.

Shibata, K., Tanuma, S., 2001. Plasmoid-induced-reconnection and fractal reconnection. Earth, Planets, and Space 53, 473-482.

Su, Y. N., Golub, L., van Ballegooijen, A. A., gros, M., 2006. Analysis of Magnetic Shear in an X17 Solar Flare on October 28, 2003. Solar Physics, 236, 325-349.

Svestka, Z., Cliver, E. W., 1992. History and Basic Characteristics of Eruptive Flares. In: Svestka, Z., Jackson, B. V., Machado, M. E. (Eds.), LNP Vol. 399: IAU Colloq. 133: Eruptive Solar Flares. pp. 1-11. 
Titov, V. S., 2005. Pinching of coronal fields. In: Reconnection of Magnetic Fields (eds. J. Birn and E.R. Priest). Cambridge University Press, Cambridge, UK, p. 5.3 .

Titov, V. S., Galsgaard, K., Neukirch, T., 2003. Magnetic Pinching of Hyperbolic Flux Tubes. I. Basic Estimations. ApJ, 582, 1172-1189.

Titov, V. S., Hornig, G., Démoulin, P., 2002. Theory of magnetic connectivity in the solar corona. JGR, 107(A8), 1164.

Titov, V. S., Priest, E. R., Démoulin, P., 1993. Conditions for the appearance of "bald patches" at the solar surface. A\&A, 276, 564-570.

Turkmani, R., Cargill, P. J., Galsgaard, K., Vlahos, L., Isliker, H., 2006. Particle acceleration in stochastic current sheets in stressed coronal active regions. A\&A, 449, 749-757.

Valori, G., Kliem, B., Keppens, R., 2005. Extrapolation of a nonlinear forcefree field containing a highly twisted magnetic loop. A\&A, 433, 335-347.

Welsch, B. T., Fisher, G. H., Abbett, W. P., Regnier, S., 2004. ILCT: Recovering Photospheric Velocities from Magnetograms by Combining the Induction Equation with Local Correlation Tracking. ApJ, 610, 1148-1156.

Wiegelmann, T., Inhester, B., Kliem, B., Valori, G., Neukirch, T., 2006. Testing non-linear force-free coronal magnetic field extrapolations with the Titov-Démoulin equilibrium. A\&A, 453, 737-741.

Williams, D. R., Török, T., Démoulin, P., van Driel-Gesztelyi, L., Kliem, B., 2005. Eruption of a Kink-unstable Filament in NOAA Active Region 10696. ApJ, 628, L163-L166.

Yokoyama, T., Shibata, K., 1994. What is the condition for fast magnetic reconnection? ApJ, 436, L197-L200.

Zwingmann, W., Schindler, K., Birn, J., 1985. On sheared magnetic field structures containing neutral points. Solar Physics, 99, 133-143. 